\title{
Free Vibration Analysis of Multi-Directional Functionally Graded Annular Plates Using the Weak Form Quadrature Element Method
}

\author{
Kuan You ${ }^{1, a}$, Hongzhi Zhong ${ }^{1, b}$ \\ 1Department of Civil Engineering, Tsinghua University, Beijing, 100084, China \\ aykce03@foxmail.com, bhzz@mail.tsinghua.edu.cn
}

\begin{abstract}
Keywords: Vibration, Multi-directional functionally graded plate, Annular plate, Weak form quadrature element method.

Abstract. Free vibration analysis of multi-directional functionally graded annular plates is achieved in this paper. Uni-directional and bi-directional exponentially varying material properties through the thickness and along the radial direction of the plate are assumed. A four variable refined plate theory and the weak form quadrature element method are employed to establish the formulation. Numerical examples are given and results are compared with other available solutions. Good agreement is achieved, demonstrating the accuracy and high convergence rate of the present method in free vibration analysis of multi-directional functionally graded annular plates.
\end{abstract}

\section{Introduction}

Functionally graded materials (FGMs), fabricated by varying percentages of two or more different material ingredients, are of high performance. The composition and structure of FGMs are in accordance with continuous variation of the specified gradient from one surface to another such that the materials can exhibit the desired property gradation in spatial directions. FGMs have shown great mechanical and thermodynamical properties in a variety of engineering applications since the end of the 20th century, especially in the field of spacecraft, optoelectronics, nuclear reactors and other high temperature situations.

A four variable refined plate theory (RPT) developed by Shimpi [1] is used in this paper. The unique feature of this theory is that the lateral displacement is assumed to be composed of two components (bending component and shear component). Unlike Reissner-Mindlin's theory, the RPT does not require the shear correction coefficient, and has strong similarities with the CPT in some aspects such as the forms of equations and expressions. It is noted that the RPT involves only two unknown variables as against five variables in the case of FSDT and HSDT. In recent years, research work about the RPT has been extended to laminated composite plates [2][3] and functionally graded plates [4][5][6].

The research on functionally graded materials has been very extensive in recent years; however, the material properties are almost exclusively assumed to vary through the thickness in the literature. Fabrication of engineering components with variable properties in two or more directions is a novel idea of using FGMs. Thus, it is necessary to develop appropriate methods to investigate the behavior of multi-directional functionally graded structures. Dynamic analysis of multi-directional functionally graded annular plates was once studied by Nie and Zhong [7] using the state space-based differential quadrature method. Free vibration and vibrational displacements of two-dimensional functionally graded fiber-reinforced curved panels was investigated by Sobhani Aragh et al. [8] The 3-D free vibration analysis of a multi-directional functionally graded piezoelectric (FGP) annular plate resting on two parameter (Pasternak) elastic foundations was investigated by Yas and Moloudi [9].

In the above mentioned papers, semi-analytical methods and differential quadrature methods are used, which are often difficult to be extended to vibration analysis of plates of FGMs in general conditions despite having available satisfactory solutions for some specific problems. In this paper, the weak form quadrature element method (QEM), a more general approach proposed by Zhong and his colleagues [10][11], is used, which has shown great adaptability and efficiency when dealing with problems with complex geometric shapes, loading conditions or non-homogeneous materials 
[12][13]. The annular plate is mapped to standard plane domain by geometric transformation, and the formulations are established in plane Cartesian coordinate system.

\section{Formulations}

The four variable refined plate theory takes into account the transverse shear deformation. Considering the in-plane displacements on the middle plane, the displacement fields of the four variable refined plate theory are expressed as

$$
\begin{aligned}
& u_{1}(x, y, z, t)=u(x, y, t)-z \frac{\partial w_{b}}{\partial x}+h\left[\frac{1}{4}\left(\frac{z}{h}\right)-\frac{5}{3}\left(\frac{z}{h}\right)^{3}\right] \frac{\partial w_{s}}{\partial x} \\
& u_{2}(x, y, z, t)=v(x, y, t)-z \frac{\partial w_{b}}{\partial y}+h\left[\frac{1}{4}\left(\frac{z}{h}\right)-\frac{5}{3}\left(\frac{z}{h}\right)^{3}\right] \frac{\partial w_{s}}{\partial y} \\
& u_{3}(x, y, z, t)=w_{b}(x, y, t)+w_{s}(x, y, t)
\end{aligned}
$$

where $u_{1}, u_{2}, u_{3}$ are the total displacements in the $x, y, z$ direction; $u, v$ are the in-plate displacements on the middle plane in the $x, y$ direction; $w \mathrm{~b}$ and $w_{\mathrm{s}}$ are the bending and shear components of transverse displacements; $h$ is the thickness of the plate; and $t$ is the time.

For the multi-directional FGM, the Young's modulus and mass density are continuous functions of the coordinates and are assumed following exponent-law distributions through the thickness and along the radial direction of the plate, i.e.

$$
E(r, z)=E(0,0) e^{\lambda_{1}\left(\frac{z}{h}\right)+\lambda_{2}\left(\frac{r}{a}\right)}, \quad \rho(r, z)=\rho(0,0) e^{\lambda_{1}\left(\frac{z}{h}\right)+\lambda_{2}\left(\frac{r}{a}\right)}
$$

where $E(0,0)$ and $\rho(0,0)$ are the corresponding values at the center point of the bottom plane, $\lambda_{1}$ and $\lambda_{2}$ denote the material property graded indexes in the thickness and radial directions, respectively.

The motion equations can be derived from Hamilton's principle

$$
\delta \int_{t_{1}}^{t_{2}}(U-T) d t=0
$$

where $U$ and $T$ are the strain energy and the kinetic energy of the system, respectively.

The QEM starts with the weak form description of the problem in an integrable subdomain of the problem. The integrals and derivatives involved in the variational formulation are approximated in sequence with the help of numerical integration and the differential quadrature analog.

Lobatto quadrature is usually chosen to evaluate the integrals involved in the QEM, i.e.

$$
\int_{-1}^{1} f(\xi) d \xi=\sum_{i=1}^{N} W_{i} f\left(\xi_{i}\right)
$$

where $N$ is the number of integration points, and $W_{\mathrm{i}}$ are the weighting coefficients of the integration scheme.

The partial derivatives at an integration point are approximated by the differential quadrature analog and generalized differential quadrature analog in the QEM for continuum problems of C0 and $\mathrm{C} 1$, respectively.

It is noted that the same set of nodes is used for both numerical integrations and derivatives, which results in great simplification in calculation procedure and post processing. Moreover, high computational accuracy is achieved by high order displacement field approximation of the whole subdomain.

Finally, the elemental strain energy can be expressed as

$$
U^{(e)}=\frac{1}{2} \sum_{i=1}^{N} \sum_{j=1}^{N} W_{i} W_{j} \mathbf{d}^{(e) T} \mathbf{T}^{T} \mathbf{B}_{i j}^{T} \boldsymbol{\Lambda}_{i j}^{T} \mathbf{D}_{i j} \Lambda_{i j} \mathbf{B}_{i j} \mathbf{T} \mathbf{d}^{(e)}|\mathbf{J}|_{i j}=\frac{1}{2} \mathbf{d}^{(e) T} \mathbf{K}^{(e)} \mathbf{d}^{(e)}
$$

where $\mathbf{D}$ is the elastic matrix, $\boldsymbol{\Lambda}$ is the geometric transformation matrix, $\mathbf{B}$ is the matrix of differential quadrature analog, $\mathbf{T}$ is the coordinate transformation matrix, $\mathbf{J}$ is the Jacobian matrix, $\mathbf{d}^{(e)}$ 
is the elemental nodal displacement vector, and $\mathbf{K}^{(e)}$ is the elemental stiffness matrix. The subscript $i j$ represent the values of corresponding matrices at numerical integration point $(i, j)$.

Similar to the discretization and approximation of the strain energy, the elemental kinetic energy can be easily obtained. After summation of all element contributions, the generalized eigenvalue equations of vibration problem can be expressed as

$$
\left(\mathbf{K}-\omega^{2} \mathbf{M}\right) \overline{\mathbf{d}}=\mathbf{0}
$$

where $\overline{\mathbf{d}}$ is the global modal vector, $\mathbf{K}$ and $\mathbf{M}$ are the global stiffness matrix and the global mass matrix, and $\omega$ is the natural frequency.

\section{Examples}

Example 1. Free vibration of functionally graded annular plate $(b=0.2 \mathrm{~m}, a=1.0 \mathrm{~m}, h=0.1 \mathrm{~m})$ with the c-c boundary condition at the inner and outer circumferential edges is studied in this example. A quarter of the plate is chosen as the computational model due to its double symmetry (see Fig. 1), and all frequencies can be obtained by applying symmetrical and antisymmetric boundary conditions on edges $\mathrm{AB}$ and $\mathrm{CD}$. The material constants for the FGM plate at the center of the bottom plate are

$$
E=380 \mathrm{Gpa}, \quad \rho=3800 \mathrm{~kg} / \mathrm{m}^{3}, \quad v=0.3
$$

The non-dimensional natural frequency parameters is defined as

$$
\bar{\omega}=\omega h \sqrt{\rho / C_{11}}, \quad C_{11}=\frac{E(1-v)}{(1+v)(1-2 v)}
$$

Results of the first three non-dimensional natural frequency parameters for the conventional uni-directional FGM $\left(\lambda_{1}=1, \lambda_{2}=0\right)$ are given in Table 1 and compared with those given by Nie et al. [7] and Yas et al. [9] who used the state space based differential quadrature method in combination of 3-D elastic theory. It is noted that the present results with refined plate theory are slightly higher than those from 3-D theory. This is ascribed to the assumption of displacement field in RPT. It can be seen that better convergence rate is achieved for the present method.
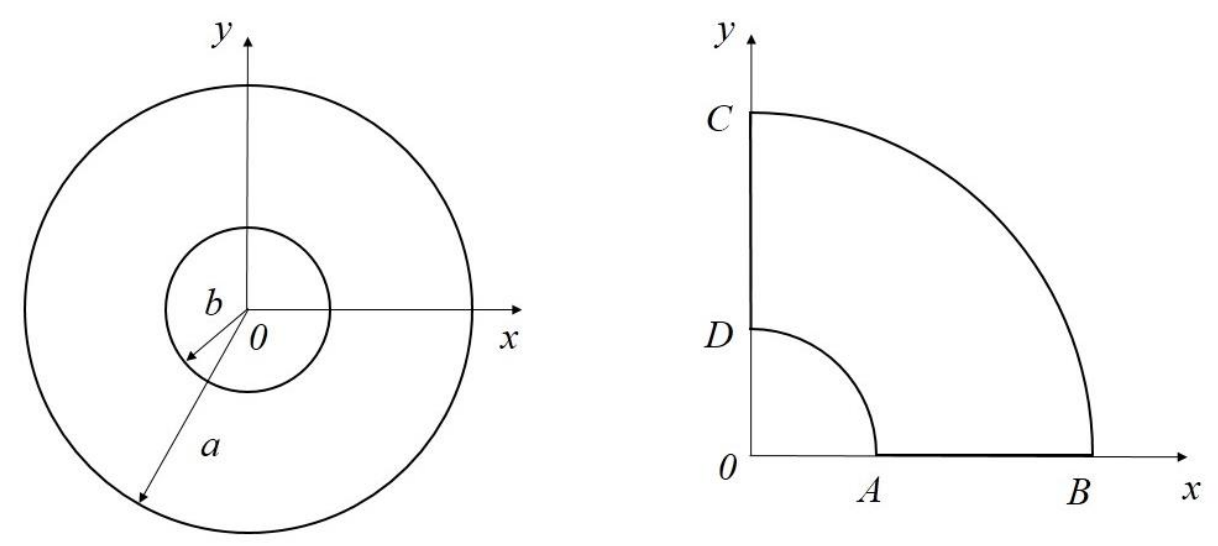

Fig. 1. A multi-directional functionally graded annular; a quarter of the plate as the computational model The comparison of the first three frequency parameters for plates of uni-directional functionally graded materials having continuous distribution along the radial direction $\left(\lambda_{1}=0, \lambda_{2}=1\right)$ and the bi-directional functionally graded materials having continuous distribution in both the radial and thickness directions $\left(\lambda_{1}=1, \lambda_{2}=1\right)$ are given in Table 2 . Good agreement is reached. 
Table 1. First three frequency parameters of functionally graded annular plate with c-c boundary conditions $\left(\lambda_{1}=1\right.$, $\left.\lambda_{2}=0\right)$.

\begin{tabular}{|c|c|c|c|c|c|}
\hline \multirow{2}{*}{\multicolumn{2}{|c|}{ Frequency }} & \multicolumn{4}{|c|}{ Number of the discrete point along the radial direction $N$} \\
\hline & & 7 & 8 & 9 & 10 \\
\hline \multirow{3}{*}{$\bar{\omega}_{1}$} & Present & 0.0815 & 0.0815 & 0.0814 & 0.0813 \\
\hline & Ref.[7] & 0.0812 & 0.0814 & 0.0802 & 0.0807 \\
\hline & Ref.[9] & 0.0799 & 0.0797 & 0.0796 & 0.0795 \\
\hline \multirow{3}{*}{$\bar{\omega}_{2}$} & Present & 0.0854 & 0.0855 & 0.0854 & 0.0853 \\
\hline & Ref.[7] & 0.0843 & 0.0843 & 0.0832 & 0.0837 \\
\hline & Ref.[9] & 0.0829 & 0.0826 & 0.0826 & 0.0825 \\
\hline \multirow{3}{*}{$\bar{\omega}_{3}$} & Present & 0.1010 & 0.1010 & 0.1009 & 0.1009 \\
\hline & Ref.[7] & 0.0969 & 0.0968 & 0.0958 & 0.0961 \\
\hline & Ref.[9] & 0.0956 & 0.0952 & 0.0952 & 0.0951 \\
\hline
\end{tabular}

Table 2. First three frequency parameters of functionally graded annular plate with c-c boundary conditions $(N=10)$.

\begin{tabular}{ccccc}
\hline \multirow{2}{*}{ Graded indexes } & \multicolumn{3}{c}{ The first three frequency parameters } \\
\cline { 3 - 5 } & & $\bar{\omega}_{1}$ & $\bar{\omega}_{2}$ & $\bar{\omega}_{3}$ \\
$\lambda_{1}=0$ & Present & 0.0836 & 0.0871 & 0.1030 \\
$\lambda_{2}=1$ & Ref.[7] & 0.0835 & 0.0870 & 0.1006 \\
& Ref.[9] & 0.0822 & 0.0856 & 0.0995 \\
$\lambda_{1}=1$ & Present & 0.0827 & 0.0868 & 0.1017 \\
$\lambda_{2}=1$ & Ref.[7] & 0.0817 & 0.0851 & 0.0985 \\
\hline
\end{tabular}

Example 2. Parametric studies for free vibration of bi-directional functionally graded annular plates are carried out for outer diameter-thickness ratio $a / h$ and inner-outer diameter ratio $b / a$. The geometry and boundary conditions are the same as those in Example 1. The variation of fundamental frequency parameter is illustrated in Fig. 2. The frequency parameter is defined as

$$
\Omega=\omega a^{2} \sqrt{\rho / C_{11}} / h
$$

It is observed in Fig. 2 that the fundamental frequency parameter increases rapidly with the increase of outer diameter-thickness ratio for $a / h<20$, and virtually reach a stable value for $a / h>30$. Meanwhile, the fundamental frequency parameter increases with the increase of inner-outer diameter ratio.
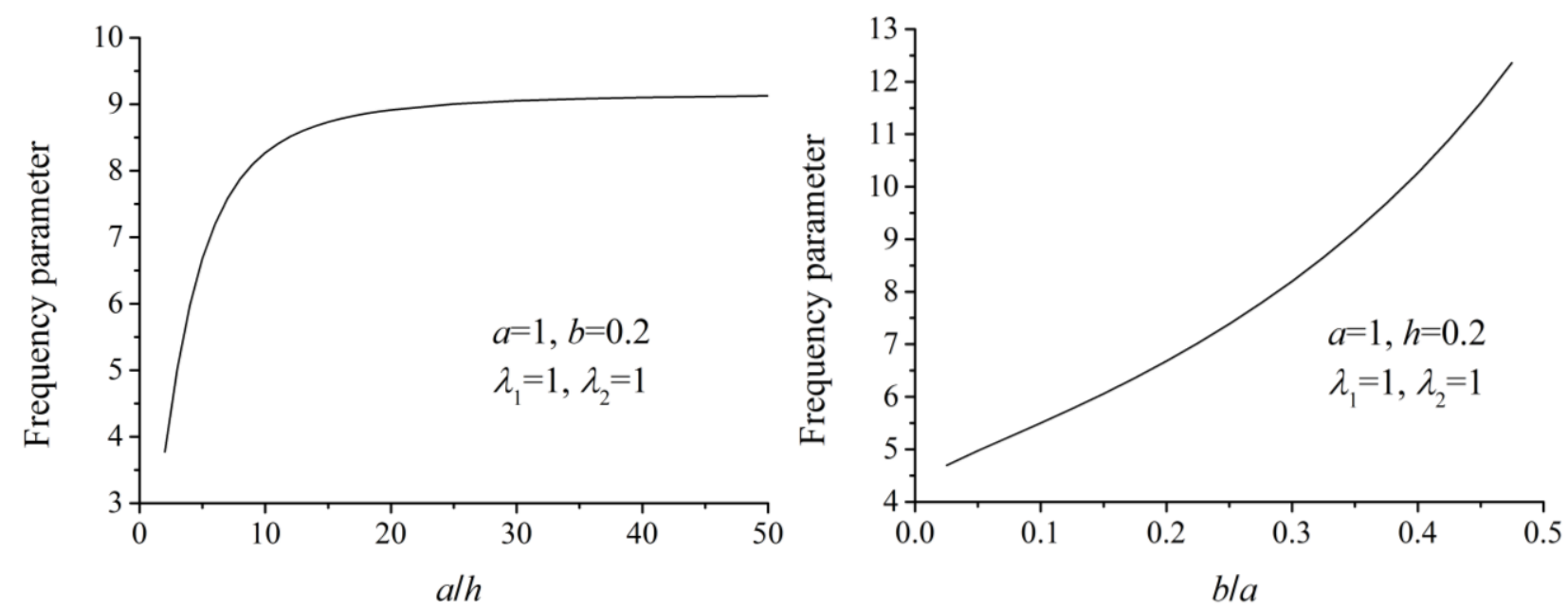

Fig. 2. (a)Variation of frequency parameter with $a / h$ ratio, (b) variation of frequency parameter with $b / a$ ratio. 


\section{Conclusions}

Free vibration analysis of multi-directional functionally graded annular plates has been conducted on the basis of the four variable refined plate theory using the weak form quadrature element method. The weak form quadrature formulations have been established in the general plane Cartesian coordinate system, enjoying good adaptability for vibration analysis of plates with arbitrary geometric shapes. Numerical examples verify the accuracy and convergence rate of the present method. Parametric studies have been carried out for free vibration of bi-directional functionally graded annular plates. It is believed that the present method can be extended to more complicated functionally graded plate problems.

\section{References}

[1] Shimpi, R. P. (2002). Refined plate theory and its variants. AIAA journal, 40(1), 137-146.

[2] Kim, S. E., Thai, H. T., \& Lee, J. (2009). A two variable refined plate theory for laminated composite plates. Composite Structures, 89(2), 197-205.

[3] Thai, H. T., \& Kim, S. E. (2010). Free vibration of laminated composite plates using two variable refined plate theory. International Journal of Mechanical Sciences, 52(4), 626-633.

[4] Mechab, I., Atmane, H. A., Tounsi, A., \& Belhadj, H. A. (2010). A two variable refined plate theory for the bending analysis of functionally graded plates. Acta Mechanica Sinica, 26(6), 941-949.

[5] Benachour, A., Tahar, H. D., Atmane, H. A., Tounsi, A., \& Ahmed, M. S. (2011). A four variable refined plate theory for free vibrations of functionally graded plates with arbitrary gradient. Composites Part B: Engineering, 42(6), 1386-1394.

[6] Bouiadjra, M. B., Ahmed Houari, M. S., \& Tounsi, A. (2012). Thermal buckling of functionally graded plates according to a four-variable refined plate theory. Journal of Thermal Stresses, 35(8), 677-694.

[7] Nie, G., \& Zhong, Z. (2010). Dynamic analysis of multi-directional functionally graded annular plates. Applied Mathematical Modelling, 34(3), 608-616.

[8] Aragh, B. S., Hedayati, H., Farahani, E. B., \& Hedayati, M. (2011). A novel 2-D six-parameter power-law distribution for free vibration and vibrational displacements of two-dimensional functionally graded fiber-reinforced curved panels. European Journal of Mechanics-A/Solids, 30(6), 865-883.

[9] Yas, M. H., \& Moloudi, N. (2015). Three-dimensional free vibration analysis of multi-directional functionally graded piezoelectric annular plates on elastic foundations via state space based differential quadrature method. Applied Mathematics and Mechanics, 36(4), 439-464.

[10]Zhong, H., \& Yu, T. (2007). Flexural vibration analysis of an eccentric annular Mindlin plate. Archive of Applied Mechanics, 77(4), 185-195.

[11]Zhong, H., \& Yue, Z. (2012). Analysis of thin plates by the weak form quadrature element method. Science China Physics, Mechanics and Astronomy, 55(5), 861-871.

[12]Zhang, R., \& Zhong, H. (2015). Weak form quadrature element analysis of geometrically exact shells. International Journal of Non-Linear Mechanics, 71, 63-71.

[13] Yuan, S., \& Zhong, H. (2014). Consolidation analysis of non-homogeneous soil by the weak form quadrature element method. Computers and Geotechnics, 62, 1-10. 